\title{
Modelling malignant progression with a finite state machine supports a two checkpoint theory of cancer
}

\author{
Robert C. Jackson
}

Pharmacometrics Ltd, Cambridge UK

\begin{abstract}
We postulate the two checkpoints theory of cancer, a model of cancer development suggesting that malignant transformation of cells requires loss of function of both the G1 checkpoint and the mitotic spindle checkpoint. Malignant progression can be described as a process analogous to a genetic algorithm, which we term the malignant progression algorithm. There are two prerequisites for this process: first, there must be competition for reproductive resources, and this is driven by loss of the G1 checkpoint; second, there must be a source of genetic variation, and this is provided by loss of the mitotic spindle checkpoint, resulting in aneuploidy. These two factors then trigger a process of Darwinian selection, driving the emergence of cells with the various abnormalities that have been termed the "hallmarks of cancer". Malignant progression is iterative, autocatalytic, and irreversible. The process can be modelled mathematically by describing the system as a finite state machine. The model indicates that loss of the two checkpoints is necessary and sufficient for tumour progression. The order of loss of the two checkpoints appears to be important: loss of the G1 checkpoint results in premalignant cells that replicate independently of physiological growth signals, but which remain diploid. Loss of the mitotic spindle checkpoint then results in aneuploid, malignant cells with highly error-prone replication, which rapidly progress to invasive, metastatic, hypoxia-tolerant, immortalised cells. This model of malignant progression has implications for the selection of anticancer drug targets and for tumour prevention strategies.
\end{abstract}

Citation: Jackson RC. Modelling malignant progression with a finite state machine supports a two checkpoint theory of cancer. Biodiscovery 2012; 1: 1; DOI: 10.7750/BioDiscovery.2012.1.1

Copyright: (C) 2012 Jackson. This is an open-access article distributed under the terms of the Creative Commons Attribution License, which permits unrestricted use, provided the original authors and source are credited.

Received: 5 June 2012; Accepted: 5 July 2012; Available online /Published: 05 July 2012

Keywords: cancer, checkpoint, finite state machine, genetic algorithm, mitosis, malignant progression

Abbreviations: $M$ checkpoint: mitotic spindle assembly checkpoint

Corresponding Author: Robert C. Jackson, e-mail: rjackson@aol.com

Conflict of Interests: No potential conflict of interest was disclosed.

\section{Introduction}

The biochemistry of induction and progression of cancer is complex and highly variable from tumour to tumour. Over 300 oncogenes and tumour suppressor genes have been documented, that is, genes that when mutated, amplified, or partially deleted are associated with malignant transformation. It has been suggested that up to six of these oncogenic mutations may be necessary for full expression of the malignant phenotype. For example,
Hanahan and Weinberg [1] describe six "hallmarks of cancer" that characterise most malignant cells: ability to proliferate in the absence of an external growth stimulus; loss of response to negative growth regulation; loss of response to physiological death signals; biochemical changes that make cells potentially immortal (e.g. expression of telomerase), loss of growth-inhibitory contact signals (resulting in invasiveness and metastasis); and ability to survive and proliferate in conditions of hypoxia. These generalisations represent a valuable 
simplification of an otherwise intractably complex pattern of changes in malignancy. One interpretation of this hypothesis is what may be termed the "restaurant menu" view of cancer: the combination of one oncogene causing enhanced cell proliferation, plus one oncogene causing loss of apoptosis, plus one oncogene causing tolerance of hypoxia, etc. will result in a fully transformed cell. This view is substantially correct, but is not necessarily the simplest interpretation of the data, and fails to capture the characteristic dynamics of malignant transformation. The resemblance of malignant progression to a process of Darwinian selection has been remarked upon by many authors (reviewed by Cahill et al. [2]). In this view, cancer is the result of progressive accumulation of somatic mutations, each of which confers a selective proliferative advantage on those cells that possess it. This undoubtedly occurs, but the dynamics of malignant progression suggests that it may not be the whole story. A number of authors have recently postulated that the essential event in malignant transformation is loss of function of the mitotic spindle assembly checkpoint [3-6]. When this checkpoint functions correctly, it acts to prevent progression to anaphase until the replicated chromosomes are correctly attached to the two centrosomes in the $\mathrm{M}$ phase cell: one member of each pair of replicated chromosomes is attached to each of the opposite spindle poles, so that each of the daughter cells receives one, and only one, of each of the pairs of replicated chromosomes. Failure of this checkpoint results in mitotic abnormalities monopolar spindles, multipolar spindles, polyploidy and aneuploidy. As pointed out by Duesberg [5], all cancers are aneuploid to some degree. Loss of control of the $\mathrm{M}$ checkpoint results in genetic variability, but the dynamics are very different from a process of accumulation of somatic mutations: the abnormalities are much more frequent, more drastic, and much more likely to result in non-viable cells, or cells that are viable but unable to replicate. However, a fraction of the aneuploid cells retain enough of the genome that they are able to replicate free of physiological constraints.

We postulate that cancer is the result of dysfunction of two cell cycle checkpoints, the retinoblastoma proteincontrolled late G1 checkpoint, and the mitotic spindle assembly checkpoint (the $\mathrm{M}$ checkpoint). Loss of control of these checkpoints has been described in cancer by many authors [3-8], and these events have been considered to be part of the multi-stage process often described as malignant progression.

In order to describe the dynamics of complex interactive systems it is usually necessary to make simplifying assumptions. An approach introduced by Von Neumann [9] was to approximate the potentially infinite number of states of such a system by a finite number of states, which may be inter-converted according to a set of transition rules, and calculating the state transitions that are assumed to occur over a series of discrete time intervals. This kind of model, termed a finite state machine [9-11] has been applied to a wide range of biological problems.

\section{Results}

The finite state machine was first used to examine the consequences of a model of malignant progression in which the transformation of a normal cell into an invasive, metastatic malignant cell proceeds by a series of four or more somatic mutations. This model of malignant progression, which we term the multistage somatic mutation hypothesis, is summarised in Figure 1. It may be described briefly as follows: Malignant progression requires multiple sequential somatic mutations. Each of these mutations confers a survival advantage on the cells carrying it, so that the proportion of cells in the total population that carries that mutation increases. When the number of cells bearing the first mutation is sufficient, there is a high likelihood that a second mutation will occur, which will confer a further selective advantage on the doubly mutant cells, whose population then rises until a third mutation becomes probable, and so on. We assume that the total number of dividing epithelial stem cells in a mouse is $1 \times 10^{7}$, that the mutation rate for somatic mutations is $3 \times 10^{-7}$ per cell division [12], that normal mouse stem cells ( $\mathrm{P} 1$ cells in Figure1) have a doubling time of $24 \mathrm{hr}[13,14]$, fully transformed cells (P5) have a doubling time of $12 \mathrm{hr}$ [15], with partially transformed cells (P2, P3, P4) having intermediate doubling times and that the life span of a mouse is 1000 days.

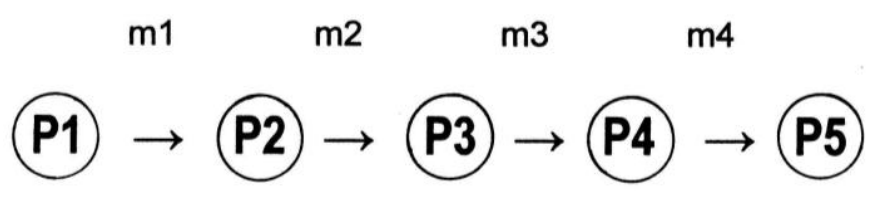

Figure 1. The four-step random order mutation model. P1 denotes normal epithelial cells and P2 - P5 denote phenotypes that cumulatively express the preceding mutations; $\mathrm{m} 1-\mathrm{m} 4$ are mutation rates.

The lower limit for macroscopic detectability of a tumour is assumed to be $10^{7}$ cells. A computer program that models the system of Figure1, termed FINITE4, is listed in the supplementary material. Running the program with the parameter values given above predicted that the lifetime probability of tumour incidence in a mouse was about $0.5 \%$. (Simulation 1: complete output from the simulations is provided in the supplementary 
information). If the assumed mutation rate was decreased to $2 \times 10^{-7}$ the predicted lifetime tumour incidence fell to less than $0.1 \%$ (Simulation 2). When the mutation rate was increased to its probable upper limit of $1 \times 10^{-6}$ the model still did not predict the appearance of a palpable tumour within the 1000 day lifetime (Simulation 3). It was concluded that the dynamics of the multistage somatic mutation model are incompatible with the observed lifetime incidence of spontaneous tumours in mice of a few percent.

The alternative theory of malignant progression, the Duesberg hypothesis [4], can be modelled by assuming that the obligatory first step is formation of an aneuploid cell (Figure 2). This has two important consequences for the daughter cells. First, a high proportion of aneuploid cells will die because they lack genes that are essential for survival. Following an unequal distribution of chromosomes at cell division, the spontaneous cell loss factor is usually at least $50 \%$, and may be greater than $90 \%$ [16]. The aneuploid cells that contain all the genes essential for survival can then go into successive cell divisions without excessive cell loss. However, since they are likely to lack one or more of the genes required for DNA proofreading, DNA repair, or accurate control of the mitotic spindle checkpoint, they tend to be genetically unstable, and effective mutation rates are now greatly increased. Modelling this situation using the program FINITE7 (Supplementary material) predicted a zero probability of tumour incidence at 1000 days (Simulation 4). P2 cells appeared, but never reached a large enough population to produce $\mathrm{P} 3$ or $\mathrm{P} 4$ cells. Because P2 cells have the same doubling time as $\mathrm{P} 1$ cells, but a greater spontaneous cell loss rate (ka), they are unable to compete with the dominant $\mathrm{P} 1$ population, and will inevitably become extinct. As Duesberg correctly points out, all cancers are aneuploid, so his theory must be essentially correct, but the modelling study shows that it cannot be the whole truth.

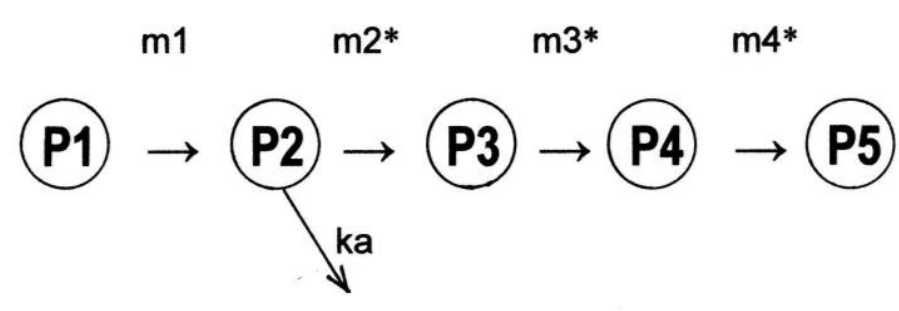

Figure 2. The Duesberg model of malignant progression; $\mathrm{m} 1$ is a mutation that causes genetic instability. ka is the rate constant for apoptosis. Other abbreviations are as defined in the legend to Figure 1. Asterisks indicate mutation rates that are modified as a result of genetic instability.
Consideration of the competition between the various normal and mutant cells suggested that a mutation that significantly decreased the cell doubling time might overcome the competitive disadvantage resulting from aneuploidy. This situation, shown in Figure 3, was modelled (Simulation 5). The first mutation, $\mathrm{m} 1$, represents loss of function of the G1 checkpoint, with a resulting change in the cell doubling time from 24 hours to 12 hours. P2 cells have a functional $\mathrm{M}$ checkpoint, so they are diploid. The second mutation, $\mathrm{m} 2$, causes defective function of the $\mathrm{M}$ checkpoint. It was assumed, initially, that the cell loss factor of $\mathrm{P} 3$ cells was 0.1 , and that mutation rates downstream from the appearance of aneuploidy were increased by 100 -fold. The model now predicted a lifetime tumour incidence in mice of 3.7\%, in rough agreement with observation. Spontaneous tumour incidence in mice varies between strains, but is typically a single-digit percentage [17]. What the model suggests, therefore, is that the development of a tumour requires loss, first of the G1 checkpoint, then of the M checkpoint, which is followed by additional mutations at a relatively high rate. I shall refer to this modified Duesberg model as the two checkpoint model. The fact that this model is consistent with observed tumour incidence in mice, but the four-stage somatic mutation model and the Duesberg model are not, is not in itself proof that the two checkpoint model is correct. However, there is a great deal of experimental evidence in support of the two checkpoint model. Cells in which the G1 checkpoint is lost but the $\mathrm{M}$ checkpoint remains intact, corresponding to $\mathrm{P} 2$ cells in figure 3 , are frequently observed. In skin, these cells form moles which are described as "premalignant". These cells are diploid, they are not invasive or metastatic, and they remain localised. Skin cells infected by human papilloma viruses (HPV) lose G1 checkpoint function, and form warts. Cells in the intestinal epithelium that lose the G1 checkpoint form intestinal polyps; again, these are diploid, and noninvasive, but are regarded as premalignant because the risk of them undergoing further mutation to a malignant growth is about a million times greater than for a normal cell. However, cells that have a defective $\mathrm{M}$ checkpoint but retain a functional G1 checkpoint have not been reported. Comparison of Simulation 4 and Simulation 5 shows that, for malignant transformation to occur, the order in which the checkpoints are lost is important. If loss of the $\mathrm{M}$ checkpoint occurs in the presence of a functional G1 checkpoint, the mutant (P2) population remains too small to progress further. This is because loss of the M checkpoint, with the resulting aneuploidy, results in a high cell loss fraction, which coupled with the unchanged doubling time, means that the $\mathrm{P} 2$ population is not self-sustaining. In contrast, when the G1 checkpoint is lost first, the cell growth rate is increased, and the $\mathrm{P} 2$ population increases rapidly. Now, when a subsequent mutation causes loss of the $\mathrm{M}$ checkpoint, the 
essentials are in place for a Darwinian selection process to occur: successful competition for reproductive resources (imposed by the limited growth surface and the rapid growth rate) and genetic variability (resulting from inaccurate chromosome segregation).

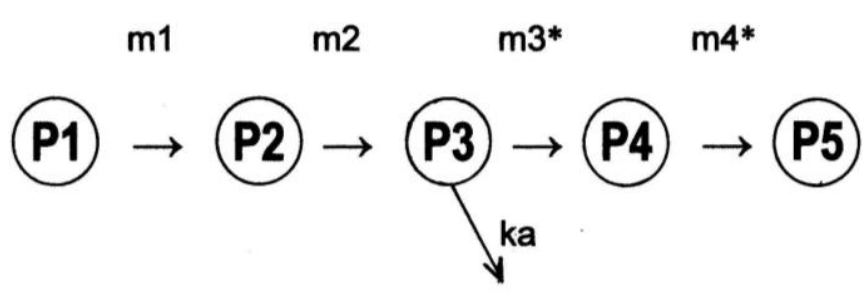

Figure 3. The two checkpoint model of malignant progression. $\mathrm{m} 1$ represents loss of $\mathrm{G} 1$ checkpoint function, and $\mathrm{m} 2$ represents loss of $\mathrm{M}$ checkpoint function. $\mathrm{P} 1-\mathrm{P} 5$ denote phenotypes as defined in Table 1. Other abbreviations are defined in the legend to Figures 1 and 2.

These calculations suggested that loss of the G1 checkpoint and of the $M$ checkpoint are necessary and sufficient for neoplastic transformation and malignant progression to occur (the two checkpoints theory of cancer). Loss of the two checkpoints enables a process of Darwinian selection in which the selective pressure is provided by competition for reproductive resources and genetic variability is provided by error-prone mitosis, a consequence of loss of the $\mathrm{M}$ checkpoint. This malignant progression algorithm (a form of genetic algorithm) is iterative, autocatalytic, and irreversible (Figure 4).

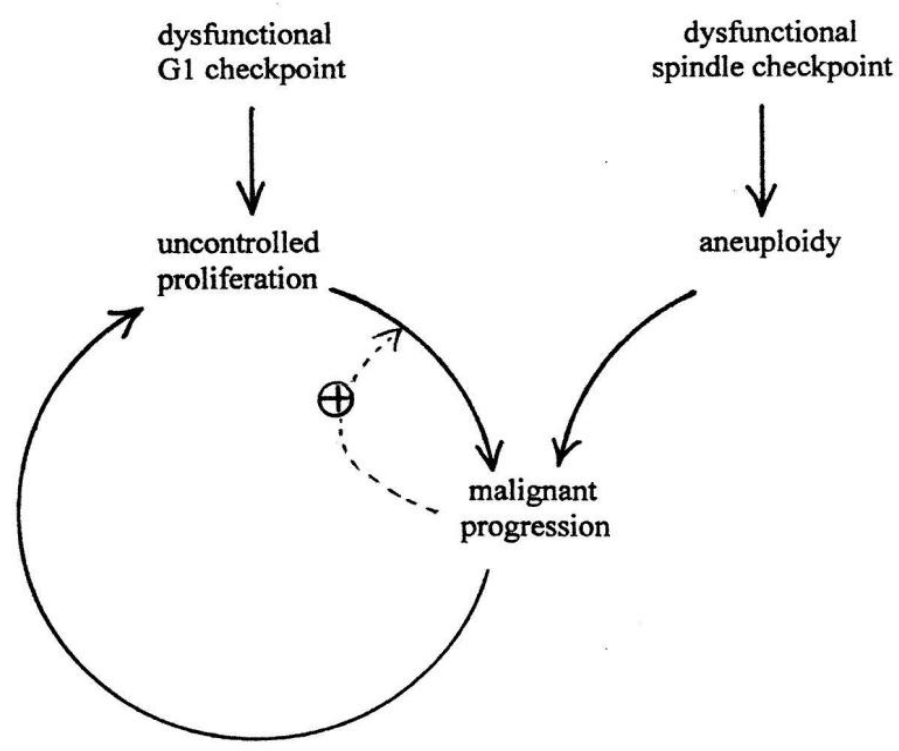

Figure 4. The malignant progression algorithm. The dashed line indicates a positive feedback loop.
By programming the malignant progression algorithm in the form of a finite state machine we can explore the consequences of varying mutation rates, selective pressure and other parameters, and elucidate the complex dynamics of malignant progression.

The malignant progression process is parameterdependent. A half-log increase in the mutation rate for loss of G1 checkpoint function resulted in a three-fold increase in the predicted tumour incidence (Simulation 6). A half-log increase in the mutation rate $m 3$ (the frequency of mutations downstream from loss of the $\mathrm{M}$ checkpoint) resulted in a large increase of predicted tumour incidence, with all mice predicted to develop tumours by the age of 26 months (Simulation 7). When the cell loss factor of $\mathrm{M}$ checkpoint-deficient cells was decreased by $50 \%$, the predicted lifetime probability of tumour development increased $113 \%$ (Simulation 8). When cell loss factor was increased $50 \%$, lifetime probability of tumour development decreased 37\% (Simulation 9). If the cell loss rate resulting from aneuploid cell division ( $\mathrm{ka}$ in Figures 2 and 3) passed beyond a threshold value of 0.9 , the doubly checkpointdefective cells could not sustain themselves and malignant progression did not occur (Simulation 10). This suggests that a possible approach to tumour prevention may be to identify agents that cause selective apoptosis of cells with a defective $M$ checkpoint.

In populations where the cell loss factor for aneuploid cells was assumed to have the default value the time for progression to a detectable tumour depended upon the doubling time of G1 checkpoint-deficient cells. Unlike the $\mathrm{M}$ checkpoint, which has all-or-none function, the $\mathrm{G} 1$ checkpoint may have partial loss of function (e.g. resulting from decreased expression of p16). A partially functional G1 checkpoint will result in a doubling time that is shorter than normal, but longer than that of a cell that expresses the fully transformed phenotype. If the doubling time of cells with defective G1 checkpoint function was increased from the default value of $12 \mathrm{hr}$ to $21 \mathrm{hr}$ (only slightly shorter than the $24 \mathrm{hr}$ doubling time of untransformed cells) the predicted probability of tumour occurrence was still $58 \%$ of the value with default parameters (Simulation 11). For tumour progression to occur, it is necessary for $\mathrm{G} 1$ checkpoint-defective cells to have a selective advantage over normal cells, but a quite small advantage is sufficient. The other parameter that directly affected tumour progression was the maximum domain cell count (as defined in "description of the algorithm"): when this was reduced by one-third, the predicted tumour incidence decreased by one-third (Simulation 12), and when the maximum domain cell count was increased by one-third the tumour incidence increased by one-third (Simulation 13).

In summary, five parameters determine the ability of a tumour cell population to progress: its doubling time, its cell loss factor, the mutation rates for loss of checkpoint 
function ( $\mathrm{m} 1$ and $\mathrm{m} 2$ ), and the maximum domain cell count.

We also modelled the situation where the end cells did not lose anchorage dependence (Simulation 14: modelled by setting $\mathrm{m} 3$ and $\mathrm{m} 4$ to zero). The eventual proportion of $\mathrm{P} 3$ cells (which are transformed but anchorage dependent) will depend upon the doubling time and the cells' loss factor, in comparison with the other cell types. Eventually, one cell type will dominate the population, but so long as the cells are limited to growth on basement membrane, they do not constitute a malignant, invasive tumour. Tumour cells that retain anchorage dependence are regarded as the earliest transformed cells in the lineage of a tumour, and are termed "cancer stem cells". These cells may be neardiploid but they are genetically unstable. However, without further mutations they do not form tumour growths. They remain in the place where they originated. In the case of epithelial tumours (such as skin cancers, and cancers of the breast, lung and colon) they need to be attached to basement membrane in order to survive. Adhesion of normal cells (or tumour stem cells) and basement membrane is complex, involving several families of cell surface receptors and associated signalling pathways. The predominant family of adhesion molecules involved in basement membrane attachment are known as integrins. If a mutation occurs in an integrin molecule or (more commonly) its associated signalling pathway ( $\mathrm{m} 3$ in Figure 3), a tumour stem cell may become able to survive without attachment to basement membrane. Normal epithelial cells that lose basement membrane attachment will die, because their survival signals require integrin signalling. A tumour stem cell that can survive without membrane attachment, no longer has to grow as a flat sheet, but can grow into a three-dimensional lump. It is now said to be an invasive tumour. These cells are shown as $\mathrm{P} 4$ in Figure 3. Such tumours are usually curable by surgery, because although they may be invasive they remain localised at or near their site of origin.

There are other families of adhesion molecules that are involved in attachments between cells. The predominant family of cell-cell adhesion molecules are the cadherins. If cadherin signalling becomes nonfunctional (mutation $\mathrm{m} 4$ in Figure 3) the tumour cells can now survive without cell-cell attachment (P5 in Figure 3). These cells are now able to detach from the tumour mass, and may move to other parts of the body in the bloodstream or the lymphatic system, and give rise to secondary growths at distant sites. P5 cells are said to be metastatic tumour cells.

Spontaneous tumours occur in mice, but as in most short-lived species, they are comparatively rare. However, in transgenic mice carrying a mutation that disrupts or over-rides the G1 checkpoint, such as a constitutively activated H-ras [18], the so-called
Oncomouse ${ }^{\mathrm{TM}}$, the incidence of tumours may approach $100 \%$. Evidently loss of the G1 checkpoint means that subsequent tumour development, while still a stochastic process, is much more common. We have modelled the situation where the first mutation is assumed to have already occurred by starting with all cells possessing the P2 phenotype, and using default cell doubling times, cell loss factors, and mutation rates to predict the rate of progression of such cells to a fully malignant phenotype. The model predicted that all mice would have macroscopically detectable tumours by the age of 22 months (Simulation 15).

Simulation 16 models the situation where anchoragedependence may be lost as a result of a single mutation, in cells that retain a functional $\mathrm{M}$ checkpoint (i.e. in diploid cells.) The calculations predicted that this situation should result in formation of a large number of diploid metastatic tumours within 90 days. Diploid metastatic tumours are never observed in nature, suggesting that simulation 16 was making incorrect assumptions. If we assume that complete loss of anchorage-dependence required two (or more) mutations, no tumours are seen when loss of anchorage-dependence precedes loss of the $\mathrm{M}$ checkpoint (Simulation 17). If we revert to the situation where loss of anchoragedependence required two mutations to occur after loss of the $\mathrm{M}$ checkpoint (Simulation 18), the situation is once again that of Figure 3, and the lifetime cancer risk in a mouse is again predicted to be about $4 \%$.

\section{Discussion}

Previous discussions of tumour progression as a process of Darwinian selection [2] have emphasized the progressive accumulation of somatic mutations, each conferring a selective advantage to the progressively more malignant cells, and culminating in the full-blown malignant phenotype, bearing all the hallmarks of cancer [1]. These events do indeed occur, but this description fails to capture the essential dynamics of the process. Not all somatic mutations are equal in their effect, and mutations that result in a dysfunctional $\mathrm{M}$ checkpoint, in particular, cause a qualitative change in the dynamics of the cell population: cells can no longer segregate their replicated chromosomes faithfully, so that the overall rate of genetic change increases abruptly. Many of the resulting aneuploid cells will no longer have all the genes necessary for survival, so the cell loss factor will show an abrupt increase. This may result in the aberrant population becoming extinct, or remaining at too low a level for a tumour to form. However, some fraction of the aneuploid cells may survive and replicate. Since these cells, by definition, have all the essential housekeeping genes, aneuploid cells after the first generation may have a cell loss factor in the normal range. At this stage, another qualitative change may 
occur: one or more of the replicating aneuploid cells may lose its susceptibility to contact inhibition, so that the cells become invasive.

For these events to happen with sufficient frequency to generate a tumour, the population of aneuploid cells must be large enough that, despite high cell loss and the low frequency of the mutation leading to loss of contact inhibition, one or more cells bearing this mutation will survive and replicate. This will only occur if the initial, contact-inhibited aneuploid cells have a selective advantage over their diploid precursors. This is why loss of the G1 checkpoint must precede loss of the M checkpoint, why a premalignant stage must precede full malignancy: to establish a critical mass of premalignant, checkpoint-defective cells, so that the product of the probability of the mutation resulting in a non-contactinhibited cell and the size of the population of cells at risk becomes great enough to overcome the unfavourable population dynamics (Figure 5). This interpretation of the required sequence of checkpoint loss is supported by the fact that while premalignant lesions $\left(\mathrm{G}^{-} \mathrm{M}+\right.$ ) are common, there are no reports of aneuploid tumours that have an intact $\mathrm{G} 1$ checkpoint $\left(\mathrm{G}+\mathrm{M}^{-}\right)$. The finite state machine predicted that $\mathrm{M}$ checkpoint deletion in absence of G1 checkpoint dysfunction could in principle result in tumour progression, but only at combinations of high mutation rate and low cell loss factor unlikely to be encountered in practice.

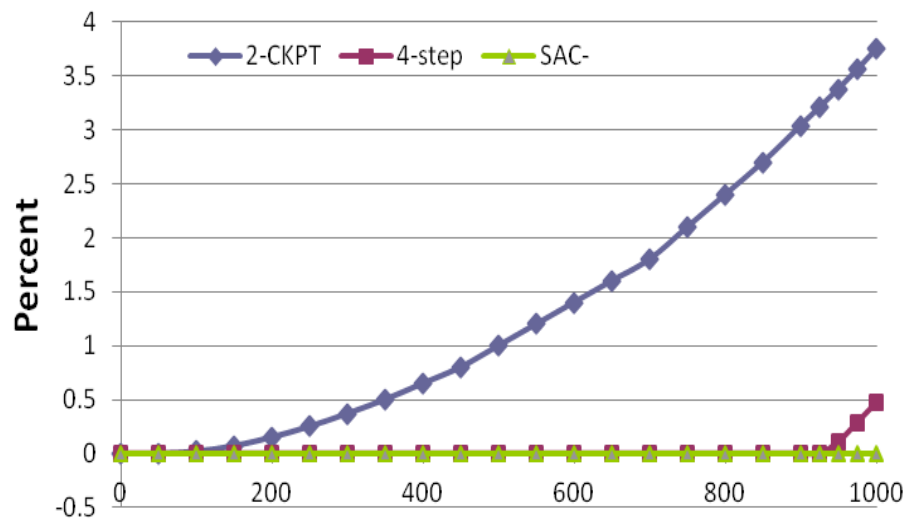

Time (days)

Figure 5. Summary of simulations 1 (4-step), 4 (SAC-) and 5 (2CKPT). The Y-axis shows the cumulative probability of tumour formation. The model in which M-checkpoint activity is lost first (SAC-) shows zero probability of tumour formation in 1000 days. For the 4-step somatic mutation model the predicted probability was $0.47 \%$, and for the 2 -checkpoint model it was $3.7 \%$.

All these features are captured in our malignant progression algorithm (Figure 4). The algorithm resembles a classical genetic algorithm [19-21] in requiring competition for reproductive resources (a "struggle for survival", in Darwinian terms), which in the case of a premalignant tumour is imposed by limited space to grow, often imposed by contact inhibition, by hydrostatic pressure, or by the requirement of epithelial cells for basement membrane contact. Loss of the G1 checkpoint provides the selective advantage that enables premalignant cells to compete for these resources. As in the classical genetic algorithm, the second prerequisite for natural selection to occur is a source of genetic variability (Darwin's "descent with modification"). Loss of the $\mathrm{M}$ checkpoint provides an abrupt and frequent source of genetic variability, albeit at the cost of an increased frequency of spontaneous cell death. These two changes set in train the process of Darwinian selection. As in the genetic algorithm, this is an iterative process. Whereas in evolution the iterations occur with the frequency of the organism's reproductive cycle, in the malignant progression algorithm, the iterations have the frequency of the cell division cycle. As with the genetic algorithm, the malignant progression algorithm is autocatalytic: with each successive iteration, the proportion of transformed cells in the population increases. Not only that, but the selection process will automatically decrease the cell loss fraction of cells undergoing spontaneous apoptosis.

It must be emphasised that the G1 checkpoint is complex, and has multiple functions: control of progression into $\mathrm{S}$ phase in response to growth factors, determination of whether a cell in G1 is destined to proliferate, remain static, enter apoptosis, or senesce, and responding to DNA damage by entering cell cycle arrest until the damaged DNA is repaired (or failing that, to undergo apoptosis). Although we argue here that all tumour cells have a dysfunctional G1 checkpoint, this does not necessarily mean that all these functions are lost. Unlike the M checkpoint [22], loss of function of the $\mathrm{G} 1$ checkpoint is not all-or-none. It is known that about $50 \%$ of human tumours retain the ability to enter cell cycle arrest following DNA damage. In the present discussion, by "dysfunctional" we mean the loss of ability to arrest in the absence of external growth factor stimulation (thereby developing a growth advantage over cells with an intact G1 checkpoint). The presence of a constitutively activated ras gene provides an example of G1 checkpoint over-ride, resulting in greatly increased spontaneous tumour incidence (Figure 6).

These then are the defining characteristics of the malignant progression algorithm: loss of the G1 checkpoint provides a competitive survival advantage; loss of the $\mathrm{M}$ checkpoint provides the required genetic variability; Darwinian selection results and increases the proportion of transformed cells in the total cell population. The process is iterative, autocatalytic, and irreversible. The order of loss of the two checkpoints is essentially obligatory, and is determined by the population dynamics of the system. In all these 


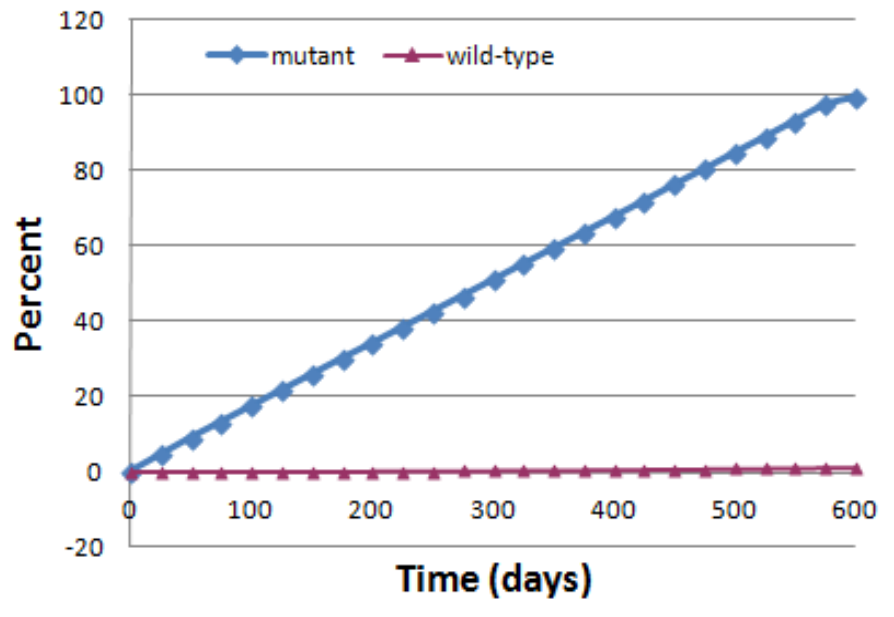

Figure 6. Predicted cumulative tumour incidence in mice with constitutively activated and wild-type ras genes calculated by the 2checkpoint model. Data plotted from simulations 5 and 15.

characteristics, malignant progression follows a classical genetic algorithm. However, it differs in one important respect. As usually implemented, genetic algorithms have a fixed objective function, so the system evolves to improve the goodness of fit (Darwinian "fitness"). In the malignant progression algorithm, cells are initially selected to give maximal proliferation within the constraints imposed upon normal cells (e.g. fixed growth area), but following loss of the $\mathrm{M}$ checkpoint and resulting loss of anchorage dependence, this constraint is removed, and cells are free to invade other spaces - in other words, the objective function has now changed. The process thus falls into two stages, selection for resources within the constraint of anchorage dependence, and unrestrained proliferation once that constraint is removed.

In this sense, the malignant progression algorithm reproduces in microcosm certain aspects of the process of evolution. Species that are optimally adapted to their environment appear through natural selection. However, if the environmental constraints change, perhaps because of a change in climate, or food availability, or because the species extends its geographic range, the constraints alter, and the selection process may now favour different genetic variants that have an advantage under the new conditions. This is the basis of speciation, and the second phase of malignant progression, in which a tumour becomes invasive and metastatic, appears to follow similar dynamics. The origin of species by natural selection has been described as an algorithm [23] and has been the topic of extensive modelling studies [24]. It may be that certain aspects of evolutionary biology could usefully be studied by finite state systems in which the switch from adaptation to speciation is modelled by a change in the objective function of the genetic algorithm.

Our studies have modelled the situation where normal and premalignant cells are restricted to growth on basement membrane, and must compete for space. This describes the kinetics of many epithelial tissues. It will be interesting to model other kinds of tissue kinetics, for example, the situation in intestinal villi, where cells originate in the crypts, and progress through a finite number of divisions (moving along the villi as they do so) and are finally sloughed into the gut lumen [25]. Our model can be used to study how tumour cell dynamics are influenced by the underlying cytokinetics of their tissues of origin.

The two checkpoints theory of cancer has implications for selection of anticancer drug targets. Given that the multiple routes through the malignant progression process first diverge, then converge, are targets early or late in the progression cascade likely to lead to broader-spectrum drugs than targets in the middle of the process? Given the pivotal role of the M checkpoint in tumour progression, will drugs that act on this checkpoint, e.g. inhibitors of aurora kinase B [22], have particularly favourable pharmacodynamics?

The two checkpoints theory also has clear implications for cancer prevention strategies. Mutations to pre-malignancy are essentially inevitable. They can be increased (e.g. by X-irradiation or ultraviolet radiation exposure) but not decreased. In contrast, it may be possible to find pharmacological approaches to minimise the progression process. The computational approach used in the present study can be extended to explore ways of doing this.

\section{Description of the algorithm}

Depending upon the status of the two checkpoints, we consider cells as having one of four genotypes: $\mathrm{G}^{+} \mathrm{M}^{+}$, $\mathrm{G}^{-} \mathrm{M}^{+}, \mathrm{G}^{+} \mathrm{M}^{-}$or $\mathrm{G}^{-} \mathrm{M}^{-}$. $\mathrm{G}^{+} \mathrm{M}^{+}$cells, with both checkpoints fully functional, are considered to be normal cells. $\mathrm{G}^{+} \mathrm{M}^{-}$cells and $\mathrm{G}^{-} \mathrm{M}^{-}$cells, with a dysfunctional $\mathrm{M}$ checkpoint, are cancer cells [5]. $\mathrm{G}^{-} \mathrm{M}^{+}$cells, lacking a functional $\mathrm{G} 1$ checkpoint, but with a normal $\mathrm{M}$ checkpoint, are pre-malignant, that is they are able to proliferate free of physiological controls, but they are non-invasive and non-metastatic.

$\mathrm{G}+$ cells are assumed to have a fixed doubling time ( $24 \mathrm{hr}$ for the purpose of our model). $\mathrm{G}^{-}$cells may have different doubling times, but these will be the same as for $\mathrm{G}+$ cells, or faster, because loss of $\mathrm{G}$ checkpoint function cannot slow down the cellular growth rate. For the purpose of most of our simulations we make the simplifying assumption that $\mathrm{G}^{-}$cells have a doubling time of $12 \mathrm{hr}$. However, so long as the $\mathrm{G}^{-}$cells have a selective advantage over G+ cells, however slight, the overall dynamics of the system do not change.

$\mathrm{M}^{+}$cells are assumed to replicate faithfully: their daughter cells will have the same genotype and 
phenotype as the parent cell except for the rare occasions (about one in every few million cell divisions) when a somatic mutation occurs. $\mathrm{M}^{+}$cells are assumed to be anchorage-dependent and contact-inhibited: they will replicate until the space available is fully occupied, and then stop replicating. $\mathrm{M}^{-}$cells have a finite probability (here termed $\mathrm{m} 3$ ) of changing at each cell division. The possible changes are loss of anchorage dependence and more rapid doubling time. In addition, some fraction, ka, of $\mathrm{M}$ checkpoint-deficient cells is assumed to undergo apoptosis; those cells that survive the first doubling may then be apoptosis-resistant. However, cells that have lost anchorage-dependence and enter the circulation are subject to destruction by various mechanisms (e.g. NK cell-mediated cyctotoxicity) so the overall cell loss rate may increase at this stage.

According to this scheme, the four genotypes can have one of 7 phenotypes (states) as follows, where DT = doubling time in hours, $\mathrm{A}=$ anchorage dependence, + or - , and where cells that have lost basement membrane dependence may or may not retain cell-cell contactdependence (Table 1). These seven phenotypes constitute the states of the finite state machine. Not all seven phenotypes seem to occur naturally: those labelled P6 and P7 have combinations of mutations that are theoretically possible but do not seem to be observed in practice. Those phenotypes that are found naturally are labelled P1 to P5 in Table 1. P1 cells are normal, and P2 cells are pre-malignant - i.e. they have a dysfunctional G1 checkpoint, but they are diploid. P3 cells are tumour stem cells: they lack both the G1 checkpoint and the M checkpoint, but are otherwise minimally transformed. P4 cells are aneuploid and have lost attachmentdependence: they are invasive but not metastatic. P5 cells are aneuploid, have acquired additional mutations, and show the fully malignant phenotype, i.e. they are invasive and metastatic.
The possible transitions between these cell types, with their associated probabilities, are shown in Figures $1-3$.

\section{Transition rules}

The number of cells in populations $\mathrm{P} 1, \mathrm{P} 2$.. $\mathrm{P} 5$ at time $\mathrm{t}$ are $\mathrm{N} 1_{(\mathrm{t})}, \mathrm{N} 2_{(\mathrm{t})}, . . \mathrm{N} 5_{(\mathrm{t})}$. Start with $\mathrm{N} 1(0)$ cells in population P1 at time zero and other populations at zero (i.e. simulations start with all normal cells). At discrete time intervals, calculate the number of new cells in each population from the previous cell number, time interval, and doubling time. From the number of doublings, and the transition probabilities (mutation rates) calculate the population transitions:

$\Delta \mathrm{P} 1=\mathrm{P} 1 *-\mathrm{m} 1$

$\Delta \mathrm{P} 2=\mathrm{P} 1 * \mathrm{~m} 1-\mathrm{P} 2 * \mathrm{~m} 2$

$\Delta \mathrm{P} 3=\mathrm{P} 2 * \mathrm{~m} 2-\mathrm{P} 3 *(\mathrm{~m} 3+\mathrm{ka})$

$\Delta \mathrm{P} 4=\mathrm{P} 3 * \mathrm{~m} 3-\mathrm{P} 4 * \mathrm{~m} 4$

$\Delta \mathrm{P} 5=\mathrm{P} 4{ }^{*} \mathrm{~m} 4$

\section{Cell domains}

The genetic algorithm assumes that cells compete for resources, but a particular cell does not compete with every other cell in the entire body. The area within which competition takes place is geographically restricted. Thus, when a skin stem cell is infected by papilloma virus, its $\mathrm{G} 1$ checkpoint is overridden and a wart develops. However, it does not cover the whole skin surface: the growth of the wart is limited to the area fed by a single afferent capillary. This area will typically contain from less than one hundred to a few hundred stem cells. We shall refer to this area of stem cells

Table 1: States of the finite state machine

\begin{tabular}{|c|c|c|c|c|c|}
\hline & Genotype & Possible phenotypes & Ploidy & $\begin{array}{l}\text { Cell-cell Contact- } \\
\text { Dependent? }\end{array}$ & $\begin{array}{c}\text { Normal or } \\
\text { transformed? }\end{array}$ \\
\hline P1 & $\mathrm{G}+\mathrm{M}+$ & $\mathrm{DT}=24, \mathrm{~A}+$ & diploid & yes & normal \\
\hline P2 & G-M+ & $\mathrm{DT}=12, \mathrm{~A}+$ & diploid & yes & premalignant \\
\hline P3 & G-M- & $\mathrm{DT}=12, \mathrm{~A}+$ & aneuploid & yes & malignant \\
\hline P4 & " & $\mathrm{DT}=12, \mathrm{~A}^{-}$ & aneuploid & yes & malignant \\
\hline P5 & “ & $\mathrm{DT}=12, \mathrm{~A}^{-}$ & aneuploidy & no & malignant \\
\hline P6 & $\mathrm{G}+\mathrm{M}^{-}$ & $\mathrm{DT}=24, \mathrm{~A}+$ & aneuploid & $?$ & malignant \\
\hline P7 & " & $\mathrm{DT}=24, \mathrm{~A}^{-}$ & aneuploid & $?$ & malignant \\
\hline
\end{tabular}


Table 2. Default parameter values for the model

\begin{tabular}{lll}
\hline Parameter & Abbreviation & Default value \\
& & \\
\hline Doubling time for normal cells & DT[1] & $24 \mathrm{hr}$ \\
\hline Doubling time for transformed cells & DT[2] & $12 \mathrm{hr}$ \\
\hline Asymptotic normal cell count & AP & 150 per domain \\
\hline Tumour evaluation size & EV & $1 \times 10^{7}$ cells \\
\hline Mutation rates for diploid cells & $\mathrm{m} 1, \mathrm{~m} 2, \mathrm{~m} 3, \mathrm{~m} 4$ & $3 \times 10^{-7}$ per cell division \\
\hline Mutation rate for aneuploid cells & $\mathrm{m2} 2^{*}, \mathrm{~m}^{*}, \mathrm{~m} 4^{*}$ & $3.0 \times 10^{-5}$ per cell division \\
\hline Rate constant for apoptosis of aneuploid cells & $\mathrm{ka}$ & 0.1 \\
\hline
\end{tabular}

(within which competition for space and for nutrients takes place) as a domain. Similarly, it is estimated that a single intestinal crypt, fed by a single afferent capillary, contains about sixty stem cells [25].

\section{The objective function:}

If the total number of anchorage-dependent cells, $\mathrm{C}_{\text {total }}$ $=\mathrm{N} 1+\mathrm{N} 2+\mathrm{N} 3$, is greater than its allowed maximum (AP), the populations of $\mathrm{P} 1, \mathrm{P} 2$ and $\mathrm{P} 3$ are reduced in proportion:

$\mathrm{N}(\mathrm{i})_{t+1}=\mathrm{N}(\mathrm{i})_{, t} / \mathrm{C}_{\text {total }} \times \mathrm{AP}$

There is no maximum permitted cell number for noncontact-inhibited cells.

Default parameter values were as shown in Table 2.

\section{Computer programs}

The programs used to run simulations $1-18$ are listed in the Supplementary Information. An outline of the algorithm is given in the appendix.

\section{Appendix}

The Genetic Algorithm implemented as a Finite State Machine

Initialise cell populations $\mathrm{P} 1$ to $\mathrm{P} 6$ and total cell number

Calculate proliferation factors for $\mathrm{P} 1$ to $\mathrm{P} 6$
Begin iterative loop

Calculate population transitions from cell numbers and mutation rates

Update population numbers to allow for cell transitions

\section{Calculate proliferation of $\mathrm{P} 1$ to $\mathrm{P} 6$}

Call objective function, and re-proportion cell numbers

$$
\downarrow
$$

If tumour size > evaluation size, exit; otherwise repeat loop.

\section{Acknowledgements}

The author thanks Dr Fordyce Davidson, Division of Mathematics, University of Dundee, for helpful advice and discussions. 


\section{References}

1. Hanahan D, Weinberg RA.. The Hallmarks of Cancer. Cell 2000; 100: 57-70.

2. Cahill DP, Kinzler KW, Vogelstein B, Lengauer C. Genetic instability and Darwinian selection in tumours. Trends in Genetics 1999; 15: M57-M60.

3. Kops GJ, Weaver BA, Cleveland DW. On the road to cancer: aneuploidy and the mitotic checkpoint. Nature Rev Cancer 2005; 5: 773-785.

4. Duesberg P, Li R, Fabarius A, Hehlann R. Aneuploidy and cancer: from correlation to causation. Contrib Microbiol 2006; 13: 16-44.

5. Duesberg P. Chromosomal chaos and cancer. Scientific American 2007; 296: 35-41.

6. Musacchio A, Salmon ED. The spindle assembly checkpoint in space and time. Nature Rev Mol Cell Biol 2007; 8: 379 393.

7. Lane DP. (Cell immortalisation and transformation by the $\mathrm{p} 53$ gene. Nature 1984; 312: 596-597.

8. Aguda BD, Tang Y. The kinetic origins of the restriction point in the mammalian cell cycle. Cell Prolif 1999; 32: 321335.

9. Von Neumann J. General and logical theory of automata, in "John von Neumann: Collected Works", (AH Taub, editor), 1963; vol. 5, pp. 288-328. New York, Pergamon Press.

10. Gill A. Introduction to the Theory of Finite-State Machines. New York, McGraw-Hill 1970.

11. Wagner F, Schmuki R, Wagner T, Wolstenholme P. Modeling Software with Finite State Machines. Boca Raton, Florida, Auerbach Publications 2006.

12. Alon U. An Introduction to Systems Biology: Design Principles of Biological Circuits. London, Chapman \& Hall 2007.
13. Simons BD, Clevers H. Stem cell self-renewal in intestinal crypt. Exp Cell Res 2011; 317: 2719-2724.

14. Lévi F, Filipski E, Iuriski I, Li XM, Innominate P. Cross-talks between circadian timing system and cell division cycle determine cancer biology and therapeutics. Cold Spring Harb Symp Quant Biol 2007; 72: 465-475.

15. Jackson RC. The Theoretical Foundations of Cancer Chemotherapy Illustrated by Computer Models. New York, Academic Press 1992.

16. Glover DM. Mitosis in Drosophila. JCell Sci 1989; 92: 137-146.

17. Harnden DG in Scientific Foundations of Oncology (T Symington and RL Carter, eds), 1976, pp. 181-190. Chicago, Heinemann.

18. Hanahan D, Wagner EF, Palmiter RD. The origins of oncomice: a history of the first transgenic mice genetically engineered to develop cancer. Genes and Dev 2007; 21: 2258-2270.

19. Holland JH. Adaptation in Natural and Artificial Systems. Ann Arbor, Univ. of Michigan Press 1975.

20. Holland JH. Emergence from Chaos to Order. 258 pp. Oxford University Press 1998.

21. Mitchell M. An Introduction to Genetic Algorithms. Cambridge MA: MIT Press 1996.

22. Mistry HB, McCallum DE, Jackson RC, Chaplain MAJ, Davidson FA. Modeling the temporal evolution of the spindle assembly checkpoint and role of aurora B kinase. Proc Natl Acad Sci USA 2008; 105: 20215-20220.

23. Dennett DC. Darwin's Dangerous Idea. New York, Simon and Schuster 1995.

24. Dawkins R. The Selfish Gene (2 ${ }^{\text {nd }}$ edition). Oxford University Press 1989.

25. Snippert HJ, van der Flier LG, Sato T, van Es JH, van den Born $\mathrm{M}$, Kroon-Veenboer $\mathrm{C}$ et al.. Intestinal crypt homeostasis results from neutral competition between symmetrically dividing Lgr5 stem cells. Cell 2010; 143: 134-144. 